\title{
System Reliability Analysis Based On Weibull Distribution and Hesitant Fuzzy Set
}

\author{
Akshay Kumar \\ Department of Applied Sciences \\ Tula's Institute, The Engineering and Management College, Dehradun, Uttarakhand, India \\ Corresponding author: akshaykr1001@gmail.com \\ Mangey Ram \\ Department of Mathematics, Computer Science and Engineering \\ Graphic Era Deemed to be University, Dehradun, Uttarakhand, India \\ E-mail: drmrswami@yahoo.com
}

(Received May 19, 2018; Accepted August 12, 2018)

\begin{abstract}
This work deals with the hesitant fuzzy number and averaging operator and fuzzy reliability with the help of Weibull lifetime distribution. Fuzzy reliability function and triangular hesitant fuzzy number also computed with $\alpha$-cut set of the proposed reliability function. After applying the averaging operator of hesitant theory, the results are better than simple fuzzy. Also at last, a numerical example has been shown that how the hesitant fuzzy and $\alpha$-cut work in case of reliability theory.
\end{abstract}

Keywords- Hesitant fuzzy number, Membership function, Weibull distribution, Hesitant fuzzy, Averaging operator.

\section{Introduction}

Reliability theory plays a key role in the history of fuzzy set theory and engineering fields. In previous years many researchers studied the fuzzy and fuzzy reliability theories and applied in daily life bases. Zadeh (1965) presented the theory of fuzzy set which is a group of objects with a continuum class of membership function and these sets are characterized by its membership function, which has every membership defined between zero and one of each class. The operations such as convexity, union, complement, intersection, relation, and inclusion etc. were discussed and several properties of these concepts in the context of fuzzy sets were analyzed. Cai et al. (1993) studied the expectation of the binary state in reliability theory applied to various engineering systems. They discussed a conceptual framework of profust reliability theory on the basis of the expectation fuzzy state and the probability expectation. Authors also introduced a recursive relation among profust reliability function, profust lifetime function and profust failure rate function. With the help of profust reliability theory they computed the different characteristics of series, parallel, mixture and coherent systems. Kacprzyk and Onisawa (1995) applied characteristics of fuzzy set theory and probability theory in system reliability estimation. They analyzed various applications of the system's reliability measures like reliability and safety of the man-machine system, quality control, fault analysis, risk determination and estimation of system structure etc. They provided some basic tools for evaluating non-probabilistic aspects of uncertainty. Singer (1990) discussed the fuzzy reliability and hazard rate of fault tree by using membership function and also using AND, OR and NEG operators. Lee et al. (2008) determined the fuzzy reliability of series and parallel systems based on the level of $(1-\beta, 1-\alpha)$ interval-valued fuzzy numbers. Xia and Xu (2011) discussed the theory of fuzzy set and generalized hesitant fuzzy set theory, which is a very useful 
International Journal of Mathematical, Engineering and Management Sciences

Vol. 3, No. 4, 513-521, 2018

https://dx.doi.org/10.33889/IJMEMS.2018.3.4-037

technique for analyzing membership function of a component of some problem in real life situation. They proposed summation of series operators for the hesitant fuzzy set and discussed some basic operations and summation operators for the hesitant fuzzy set. In this study they developed a relationship between IFS and hesitant fuzzy set based on operators and decision making problems. Jamkhaneh (2011) defined lifetime distribution component on the basis of fuzzy exponential distribution and evaluated system reliability. A method has been proposed for computing fuzzy reliability function and its $\alpha$-cut sets and also discussed fuzzy reliability function for the series system, parallel system and $k$-out-of- $n$ system. He presented some motivated examples to demonstrate how to obtain fuzzy reliability and its $\alpha$-cut sets. Jamkhaneh (2012) estimated the fuzzy reliability and mean time to failure of the system with the help of fuzzy parameters techniques and also used normal, exponential and Weibull distributions. Zhang (2013) discussed the characteristic of the hesitant fuzzy set which is a useful generalization of the fuzzy set theory. Many times it is difficult to predict the membership of components of a set due to ambiguity between some different values. He developed hesitant fuzzy sets which are widely used by hesitant fuzzy power operators for summation. He introduced various power operators for summation using operators to hesitant fuzzy sets. He proposed some effective techniques for new aggregation operators with multi criteria decision making of the hesitant fuzzy set. Ram (2013) studied a survey on system reliability approaches. In the recent year reliability theory becomes the most searching and testing theory. Researchers have proposed various theories and methods of reliability evaluation such as; Bayes approach, coherent systems approach, copula approach, coverage factor approach, designed experiments, distributed system, genetic algorithm, nano and network system approach, etc. in various fields of engineering and physical sciences. Qian et al. (2013) presented the theory of hesitant fuzzy sets as a useful tool with group decision making problems especially when experts have a hesitation among several possible memberships for the component to a set. During the computing process in practice, however, these possible memberships may not be only crisp values in $[0,1]$, but also interval values. They studied hesitant fuzzy sets through intuitionistic fuzzy sets which are a generalized hesitant fuzzy set theory. They provided some basic operations of generalized hesitant fuzzy sets. With the help of score function and consistency function, they introduced the comparison between laws to distinguish two generalized hesitant fuzzy sets. Also showed that extension principle enables decision makers to apply summation operators on intuitionistic fuzzy sets to the summation of generalized hesitant fuzzy sets for decision making problem. Liu et al. (2014) evaluated system reliability and discussed randomness and fuzziness of lifetime components of unrepairable systems. They obtained mathematical expressions of a random fuzzy unrepairable system like the series system, parallel system, series-parallel systems, parallelseries systems and cold standby systems using reliable switches. At last numerical example are taken to demonstrate the evaluation of system reliability and MTTF for the random fuzzy unrepairable system. Jamkhaneh (2014) discussed the reliability characteristics of fuzzy set theory and lifetime distribution and Fuzzy Weibull distribution. Various methods for computing fuzzy reliability function, $\alpha$-cut set and fuzzy hazard rate for the series system, parallel system and complex system fuzzy functions are investigated. The procedure to obtain system fuzzy function has been described with an example. Wang and Chen (2014) discussed the wide applications of hesitant fuzzy softest in the field of multi criteria decision making and defined the basic operations such as compliment, AND, OR, union and intersection. Chaube and Singh (2016a) considered a two-stage weighted- $k$-out- $n$ system with common components for determining fuzzy reliability. They developed an algorithm for calculating fuzzy reliability and created the minimal path sets and minimal cut sets of two-stage weighted $k$-out-of- $n$ systems. On the basis of minimal cuts and minimal paths they investigated the fuzzy reliability of the systems within $s$-dependent component failures. Also described two kinds of weighted- $k$-out-of- $n$ systems series and parallel. Chaube and 
International Journal of Mathematical, Engineering and Management Sciences

Vol. 3, No. 4, 513-521, 2018

https://dx.doi.org/10.33889/IJMEMS.2018.3.4-037

Singh (2016b) developed a method for evaluating system fuzzy reliability of engineering systems. They computed fuzzy reliability on the basis of physical data of reliability and shape. Kumar et al. (2016) computed the fuzzy reliability for consecutive- $k$-out-of- $n: F$ system of non-identical components by using intuitionistic fuzzy set and supplementary technique. Xu (2016) discussed the hesitant fuzzy set theory and its operations. Hesitant fuzzy set have widely used in real life such as decision-making, medical diagnosis and information retrieval .Kumar et al. (2017) discussed the fuzzy reliability of series, parallel and consecutive- $k$-out-of- $n: F$ system of non-identical components using hesitant fuzzy set theory and aggregation operators with the help of Weibull distribution. Pramy (2018) computed the fuzzy multi-objective linear fractional programming problems from using graded mean integration representation method.

In the previous studies, many researchers discussed the fuzzy like intuitionistic fuzzy, bi fuzzy, hesitant, dual hesitant and many types of fuzzy reliability used in engineering systems and many real life problems in daily life. In this paper we have evaluated the hesitant fuzzy with membership function using Weibull distribution and also discussed the fuzzy reliability characteristic with their $\alpha$-cut set.

\section{Definitions}

\subsection{Fuzzy Set}

Zadeh (1965) first to introduce the concept of fuzzy set theory and its characteristics in case of membership and non-membership function of their $\alpha$-cut set. Let us consider a class $Y$ be the nonempty set of the universe of discourse of $Y=\left(y_{1}, y_{2}, \ldots y_{n}\right)$. A fuzzy set $Z$ is defined by its membership function $\mu_{Z}: Y \rightarrow[0,1]$, where $\mu_{Z}(Y)$ is the degree of membership component of $z$ in a fuzzy set $Y$ for all $y \in Y$.

\subsection{Intuitionistic Fuzzy Set}

Atanassov (1983) has given the new concept of set theory which is called intuitionistic fuzzy set theory. In case of the intuitionistic fuzzy set each component has both degree of membership and non-membership of their $\alpha$-cut and $\beta$-cut set. Let us $A$ be a subset of $b$ in the form of

$A=\left\{<b, \mu_{A}(b), v_{A}(b)>: a<A\right\}$

where, $\mu_{A}: b \rightarrow[0,1]$ and $v_{A}: b \rightarrow[0,1]$ with $\mu_{A}(b)+v_{A}(b) \leq 1, \forall b \in B$.

\subsection{Hesitant Fuzzy Set}

Naukara and Torra (2009) discussed the theory of hesitant fuzzy set within a fixed set $\mathrm{Y}$ in the form of a function when applied to $Y$ and back to the subset of $[0,1]$, which is

$$
B=\left\{\left(y, h_{B}(y)\right) / y \in Y\right\}
$$

where, $h_{B}(z)$ is the set of the value $[0,1]$, the component $y \in Y$ show the degree of membership of the set B and $h$ is called the hesitant fuzzy component (HFC) of the set.

Xio and $\mathrm{Xu}$ (2011) discussed some basic operations of the hesitant fuzzy component are as follows 
International Journal of Mathematical, Engineering and Management Sciences

Vol. 3, No. 4, 513-521, 2018

https://dx.doi.org/10.33889/IJMEMS.2018.3.4-037

(i) $h^{\lambda}=\bigcup_{\delta \in h}\left\{\delta^{\lambda}\right\}, \lambda>0$

(ii) $\lambda h=\bigcup_{\delta \in h}\left\{1-(1-\delta)^{\lambda}\right\}, \lambda>0$

(iii) $h_{1} \oplus h_{2}=\bigcup_{\delta_{1} \in h_{1}, \delta_{2} \in h_{2}} \operatorname{Max}\left\{\delta_{1}+\delta_{2}-\delta_{1} \delta_{2}\right\}$

(iv) $h_{1} \otimes h_{2}=\bigcup_{\delta_{1} \in h_{1}, \delta_{2} \in h_{2}} \operatorname{Min}\left\{\delta_{1} \delta_{2}\right\}$.

Some fundamental basic operations defined by Torra (2010) are

(i) $h^{c}=\bigcup_{\delta \in h}\{1-\delta\}$

(ii) $h_{1} \cup h_{2}=\bigcup_{\delta_{1} \in h_{1}, \delta_{2} \in h_{2}} \operatorname{Max}\left\{\delta_{1} \delta_{2}\right\}$

(iii) $h_{1} \cap h_{2}=\bigcup_{\delta_{1} \in h_{1}, \delta_{2} \in h_{2}} \operatorname{Min}\left\{\delta_{1} \delta_{2}\right\}$.

\subsection{Triangular Hesitant Fuzzy Set}

\section{Definition 1}

Chang (1996) has given the concept of the triangular fuzzy number in which a fuzzy number $\chi$ on real value $R$ said to be a triangular fuzzy number of the given membership function $\mu_{\chi}: R \rightarrow[0,1]$ if

$$
\mu_{\chi}(z)=\left\{\begin{array}{l}
\frac{x-\chi^{L}}{\chi^{M}-\chi^{L}}, z \in\left[\chi^{L}, \chi^{M}\right] \\
\frac{x-\chi^{U}}{\chi^{M}-\chi^{U}}, z \in\left[\chi^{M}, \chi^{U}\right] \\
0 \quad \text { otherwise }
\end{array}\right.
$$

where, $\chi^{L} \leq \chi^{M} \leq \chi^{U}$ and $\chi^{L}, \chi^{U}$ and $\chi^{M}$ defined as lower, upper and modal value of the fuzzy number $\chi$.

Let us suppose there are two triangular fuzzy numbers $\chi_{1}=\left(\chi_{1}{ }^{L}, \chi_{1}{ }^{M}, \chi_{1}^{U}\right)$ and $\chi_{2}=\left(\chi_{2}{ }^{L}, \chi_{2}{ }^{M}, \chi_{2}{ }^{U}\right)$ and these operations are as follows

(i) $\chi_{1} \oplus \chi_{2}=\left(\chi_{1}{ }^{L}, \chi_{1}{ }^{M}, \chi_{1}^{U}\right) \oplus\left(\chi_{2}{ }^{L}, \chi_{2}{ }^{M}, \chi_{2}{ }^{U}\right)=\left(\chi_{1}{ }^{L}+\chi_{2}{ }^{L}, \chi_{1}{ }^{M}+\chi_{2}{ }^{M}, \chi_{1}^{U}+\chi_{2}{ }^{U}\right)$

(ii) $\chi_{1} \otimes \chi_{2}=\left(\chi_{1}{ }^{L}, \chi_{1}{ }^{M}, \chi_{1}{ }^{U}\right) \otimes\left(\chi_{2}{ }^{L}, \chi_{2}{ }^{M}, \chi_{2}{ }^{U}\right)=\left(\chi_{1}{ }^{L} \chi_{2}{ }^{L}, \chi_{1}{ }^{M} \chi_{2}{ }^{M}, \chi_{1}{ }^{U} \chi_{2}{ }^{U}\right)$

(iii) $\chi_{1}=\chi_{2} \Leftrightarrow \chi_{1}{ }^{L}=\chi_{2}{ }^{L}, \chi_{1}{ }^{M}=\chi_{2}{ }^{M}, \chi_{1}^{U}=\chi_{2}^{U}$

(iv) $\chi_{1} \leq \chi_{2} \Leftrightarrow \chi_{1}{ }^{L} \leq \chi_{2}{ }^{L}, \chi_{1}{ }^{M} \leq \chi_{2}{ }^{M}, \chi_{1}{ }^{U} \leq \chi_{2}{ }^{U}$ 
International Journal of Mathematical, Engineering and Management Sciences

Vol. 3, No. 4, 513-521, 2018

https://dx.doi.org/10.33889/IJMEMS.2018.3.4-037

(v) $\beta \cdot \chi_{1}=(\beta, \beta, \beta)\left(\chi_{1}{ }^{L}, \chi_{1}{ }^{M}, \chi_{1}^{U}\right)=\left(\beta \chi_{1}{ }^{L}, \beta \chi_{1}{ }^{M}, \beta \chi_{1}^{U}\right), \beta>0, \beta \in R$

(vi) $\chi_{1}^{-1}=\left(\chi_{1}{ }^{L}, \chi_{1}^{M}, \chi_{1}^{U}\right)^{-1}=\left(1 / \chi_{1}^{U}, 1 / \chi_{1}^{M}, 1 / \chi_{1}^{U} 1 / \chi_{1}^{L}\right)$.

\section{Definition 2}

Let us consider $g_{i}(i=1,2, \ldots, n)$ be a group of the triangular hesitant fuzzy components $W=\left(w_{1}, w_{2}, \ldots w_{n}\right)^{t}$ is a weight vector of $g_{i}(i=1,2, \ldots, n)$ with $W_{i} \in[0,1]$ and $\sum_{i=1}^{n} W_{i}=1$, hence a triangular hesitant fuzzy weighted averaging is given as

$G^{n} \rightarrow G=\left(g_{1}, g_{2}, \ldots, g_{n}\right)=\bigotimes_{i}^{n}\left(W_{i} g_{i}\right)$

which is knows as aggregation operator for the triangular hesitant fuzzy set.

\subsection{Fuzzy Weibull Distribution}

The Weibull distribution is one of the most widely used distributions in reliability analysis. The probability density function of Weibull distribution is given by

$$
f(t)= \begin{cases}\frac{\beta}{\theta}\left(\frac{t}{\theta}\right)^{\beta-1} \exp \left\{-\left(\frac{t}{\theta}\right)^{\beta}\right\}, & x>0, \theta>0, \beta>0 . \\ 0, & \text { otherwise }\end{cases}
$$

where $\theta$ is a scale parameter and $\beta$ is a shape parameter.

By changing the value of the shape parameter, this distribution can be modeled a wide variety of lifetime data. If $\beta=1$ the Weibull distribution is reduced to an exponential distribution; if $\beta=2$, the Weibull distribution is identical to Rayleigh distribution.

The hazard function is defined as

$$
h(t)=\lim _{\Delta t \rightarrow 0} \frac{P(t<x<t+\Delta t)}{\Delta t}=\frac{f(t)}{R(t)} .
$$

If the lifetime follows the fuzzy Weibull distribution, then the fuzzy hazard function is given by $h(t)=\frac{\beta}{\theta}\left(\frac{t}{\theta}\right)^{\beta-1}$.

So, the reliability function of a component/system is obtained as follows

$$
R(t)=\int_{t}^{\infty} f(x) d x
$$


International Journal of Mathematical, Engineering and Management Sciences

Vol. 3, No. 4, 513-521, 2018

https://dx.doi.org/10.33889/IJMEMS.2018.3.4-037

$$
\begin{aligned}
& =\int_{t}^{\infty} \frac{\beta}{\theta}\left(\frac{x}{\theta}\right)^{\beta-1} \exp \left\{-\left(\frac{x}{\theta}\right)^{\beta}\right\} d x \\
& =\exp \left\{-\left(\frac{x}{\theta}\right)^{\beta}\right\}
\end{aligned}
$$

\section{Fuzzy reliability functions}

Fuzzy reliability function based on the fuzzy probability $R(t)$ of a unit survives within time $t$. Suppose a random variable $Z$ is a lifetime and probability density function $F(z, \theta)$ is the component of a system and $F_{z}(t)=p(z \leq t)$ is a fuzzy cumulative distribution function, where $\theta$ is known as the fuzzy parameter. Hence, the fuzzy reliability function at time $t$ is given as

$$
R(t)=p(z>t)=1-F_{z}(t)=\left\{1-F_{\max }(z)[\alpha], 1-F_{\min }(z)[\alpha], \mu_{F(z)}=\alpha\right\}, t>0 .
$$

Fuzzy failure probability is denoted by $q(t)$ with in time interval $[0, t]$ such that $q(t)=p(z \leq t)=F_{z}(t), \quad t>0$.

Hence, the fuzzy reliability function of a component is defined as (see Buckley, 2006; Jamkhaneh 2014).

$$
R(t)[\alpha]=\left\{\int_{t}^{\infty} \frac{\beta}{\theta}\left(\frac{z-\delta}{\theta}\right)^{\beta-1} e^{-\left(\frac{x-\delta}{\theta}\right)^{\beta}} d x \mid \theta \in \theta[\alpha]\right\}
$$

With the help of equation (5), the $\alpha$-cut of the reliability function is defined as

$$
R(t)[\alpha]=\left[\exp \left(-\left(\frac{t-\delta}{a_{1}+\left(a_{2}-a_{1}\right) \alpha}\right)^{\beta}\right), \exp \left(-\left(\frac{t-\delta}{a_{4}-\left(a_{4}-a_{3}\right) \alpha}\right)^{\beta}\right)\right.
$$

\section{Numerical}

Let us consider the lifetime component of a system with Weibull distribution to the hesitant membership function $\varphi$ are obtained and if $\varphi=\varphi_{1} ;(1.5,2,2.5)$, $\varphi_{2} ;(1.75,1.85,1.95), \varphi_{3} ;(2.25,2.5,2.75)$ which is triangular hesitant fuzzy within equal weight allocation $W_{1}=W_{2}=W_{3}=1 / 3$, then $\alpha$-cut of the membership function is defined as

$$
\begin{aligned}
& \varphi_{1}=(1.5+0.5 \alpha, 2.5-0.5 \alpha), \\
& \varphi_{2}=(1.75+0.10 \alpha, 1.95-0.10 \alpha), \\
& \varphi_{3}=(2.25+0.25 \alpha, 2.75-0.2 .5 \alpha)
\end{aligned}
$$


International Journal of Mathematical, Engineering and Management Sciences

Vol. 3, No. 4, 513-521, 2018

https://dx.doi.org/10.33889/IJMEMS.2018.3.4-037

$$
\begin{aligned}
R(t)[\alpha] & =\left[\exp \left(-\left(\frac{t}{1.5+0.5 \alpha}\right)^{\beta}\right), \exp \left(-\left(\frac{t}{2.5-0.5 \alpha}\right)^{\beta}\right)\right. \\
& \exp \left(-\left(\frac{t}{1.75+0.1 \alpha}\right)^{\beta}\right), \exp \left(-\left(\frac{t}{1.95-0.1 \alpha}\right)^{\beta}\right) \\
& \left.\exp \left(-\left(\frac{t}{2.25+0.25 \alpha}\right)^{\beta}\right), \exp \left(-\left(\frac{t}{2.75-0.25 \alpha}\right)^{\beta}\right)\right]
\end{aligned}
$$

For all $\alpha$, where taking $\beta=0.5$ and $t=5$.

In Table 1 , the $\alpha$-cut set of proposed reliability function using hesitant fuzzy and Weibull distribution has been shown based on the membership function from equation (6).

Table 1. $\alpha$-cut of the hesitant fuzzy membership function of the reliability system

\begin{tabular}{|c|c|c|c|}
\hline$\alpha$-cut & $R_{I}[\alpha]$ & $R_{2}[\alpha]$ & $R_{3}[\alpha]$ \\
\hline 0 & $(0.1611,0.2431)$ & $(0.1845,0.2016)$ & $(0.2252,0.2597)$ \\
\hline 0.1 & $(0.1660,0.2397)$ & $(0.1854,0.2008)$ & $(0.2271,0.2581)$ \\
\hline 0.2 & $(0.1707,0.2361)$ & $(0.1862,0.2000)$ & $(0.2289,0.2564)$ \\
\hline 0.3 & $(0.1754,0.2326)$ & $(0.1871,0.1991)$ & $(0.2307,0.2548)$ \\
\hline 0.4 & $(0.1800,0.2289)$ & $(0.1880,0.1983)$ & $(02326,0.2532)$ \\
\hline 0.5 & $(0.1845,0.2252)$ & $(0.1889,0.1975)$ & $(0.2343,0.2515)$ \\
\hline 0.6 & $(0.1889,0.2215)$ & $(0.1897,0.1966)$ & $(0.2361,0.2466)$ \\
\hline 0.7 & $(0.1932,0.2176)$ & $(0.1906,0.1958)$ & $(0.2379,0.2482)$ \\
\hline 0.8 & $(0.1975,0.2137)$ & $(0.1915,0.1949)$ & $(0.2397,0.2465)$ \\
\hline 0.9 & $(0.2016,0.2098)$ & $(0.1923,0.1941)$ & $(0.2414,0.2448)$ \\
\hline 1 & $(0.2057,0.2057)$ & $(0.1932,0.1932)$ & $(0.2431,0.2431)$ \\
\hline
\end{tabular}

Table 2 shows the fuzzy reliability of the system on the basis of Table 1, averaging operator with given equal weights and Weibull distribution of equation (3).

Table 2. Fuzzy reliability of the reliability function

\begin{tabular}{|l|c|}
\hline$\alpha$-cut & $R[\alpha]$ \\
\hline 0 & $(0.1907,0.2353)$ \\
\hline 0.1 & $(0.1932,0.2332)$ \\
\hline 0.2 & $(0.1956,0.2313)$ \\
\hline 0.3 & $(0.1981,0.2291)$ \\
\hline 0.4 & $(0.2006,0.2272)$ \\
\hline 0.5 & $(0.2035,0.2251)$ \\
\hline 0.6 & $(0.2050,0.2230)$ \\
\hline 0.7 & $(0.2076,0.2208)$ \\
\hline 0.8 & $(0.2098,0.2187)$ \\
\hline 0.9 & $(0.2134,0.2148)$ \\
\hline 1 & $(0.2144,0.2144)$ \\
\hline
\end{tabular}


International Journal of Mathematical, Engineering and Management Sciences

Vol. 3, No. 4, 513-521, 2018

https://dx.doi.org/10.33889/IJMEMS.2018.3.4-037

Also, Fig. 1 shows the hesitant fuzzy membership function based on reliability function by using Table 1 and averaging operator from Table 2 as given below.

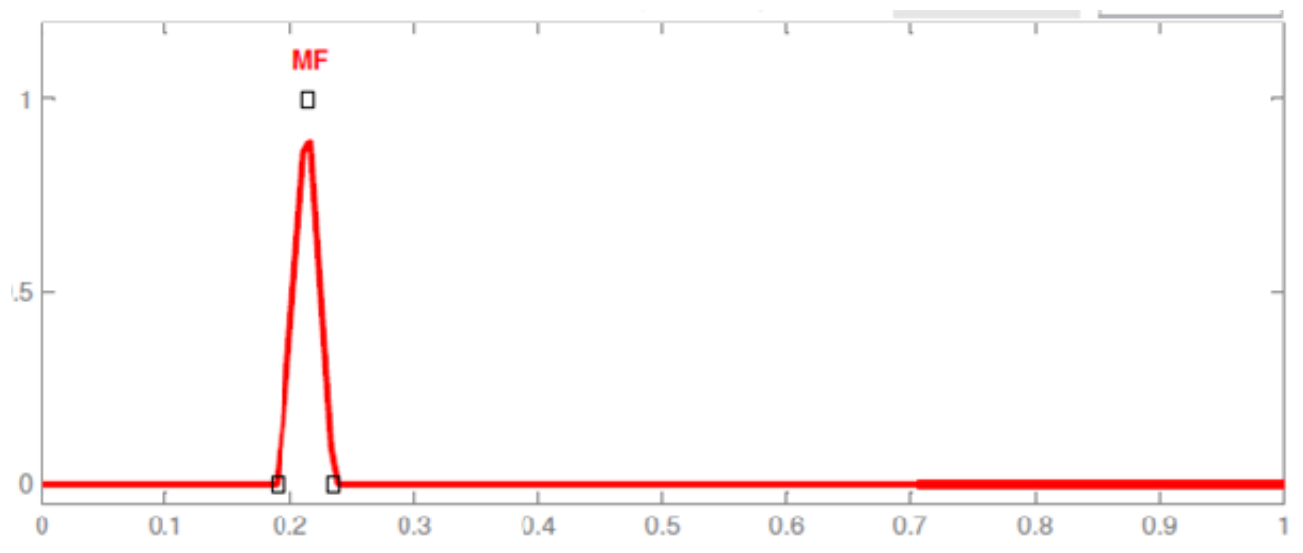

Fig 1. Fuzzy membership function

\section{Conclusion}

The results of the hesitant fuzzy number by using Weibull lifetime distribution and averaging operators have been discussed. In case of averaging operators result reached near about crisps set and better than hesitant fuzzy set. The reliability of the system with the help of hesitant fuzzy set has been evaluated and also computed the $\alpha$-cut set of the membership function. Further, the triangular hesitant fuzzy set and triangular hesitant fuzzy weighted averaging have been discussed.

\section{References}

Atanassov, K. T. (1983). Intuitionistic fuzzy sets, VI ITKR's Session, Sofia deposed in Central Sci-Technical Library of Bulg. Acad. of Sci., Bulgarian, 1697, 84.

Buckley, J. J. (2006).Fuzzy probability and statistics (223-234). Heidelberg: Springer.

Cai, K. Y., Wen, C. Y. \& Zhang, M. L. (1993). Fuzzy states as a basis for a theory of fuzzy reliability. Microelectronics Reliability, 33(15), 2253-2263.

Chang, D. Y. (1996). Applications of the extent analysis method on fuzzy AHP. European Journal of Operational Research, 95(3), 649-655.

Chaube, S., \& Singh, S. B. (2016a). Fuzzy reliability of two-stage weighted $k$-out-of- $n$ systems with common components. International Journal of Mathematical, Engineering and Management Sciences, 1(1), 4151.

Chaube, S., \& Singh, S. B. (2016b). Fuzzy reliability theory based on membership function. International Journal of Mathematical, Engineering and Management Sciences, 1(1), 34-40. 
International Journal of Mathematical, Engineering and Management Sciences

Vol. 3, No. 4, 513-521, 2018

https://dx.doi.org/10.33889/IJMEMS.2018.3.4-037

Jamkhaneh, E. B. (2011). An evaluation of the systems reliability using fuzzy lifetime distribution. Journal of Applied Mathematics, Islamic Azad University of Lahijan, 7(28), 73-80.

Jamkhaneh, E. B. (2014). Analyzing system reliability using fuzzy Weibull lifetime distribution. International Journal of Applied, 4(1), 93-102.

Jamkhaneh, E. B., (2012). Reliability estimation under the fuzzy environments. The Journal of Mathematics and Computer Science, Islamic Azad University of Lahijan, 5(1), 28-39.

Kacprzyk, J., \& Onisawa, T. (1995). Reliability and safety analyses under fuzziness, Springer PhysicaVerlag, vol.4.

Kumar A., Singh, S. B., \& Ram M. (2017). Reliability assessment using hesitant fuzzy set. International Journal of Operational Research. (Accepted)

Kumar, A., Singh, S. B., \& Ram, M. (2016). Reliability appraisal for consecutive- $k$-out-of- $n$ :F system of nonidentical components with intuitionistic fuzzy set. International Journal of Operational Research. (Accepted)

Lee, H. M., Shih, T. S., Su, J. S., \& Chen, H. S. (2008, July). Fuzzy system reliability analysis based on level $(1-\beta, 1-\alpha)$ interval-valued fuzzy numbers and using statistical data. In Machine Learning and Cybernetics, 2008 International Conference on (Vol. 7, pp. 3744-3749). IEEE.

Liu, Y., Li, X., \& Li, J. (2014). Reliability analysis of random fuzzy unrepairable systems. Discrete Dynamics in Nature and Society. Hindawi, New York, USA

Pramy, F. A. (2018). Fuzzy reliability of two-stage weighted $k$-out-of- $n$ systems with common components. International Journal of Mathematical, Engineering and Management Sciences, 3(3), 280-293.

Qian, G., Wang, H., \& Feng, X. (2013). Generalized hesitant fuzzy sets and their application in decision support system. Knowledge-Based Systems, 37, 357-365.

Ram, M. (2013). On system reliability approaches: a brief survey. International Journal of System Assurance Engineering and Management, 4(2), 101-117.

Singer, D. (1990). A fuzzy set approach to fault tree and reliability analysis. Fuzzy Sets and Systems, 34(2), 145-155.

Torra, V. (2010). Hesitant fuzzy sets. International Journal of Intelligent Systems, 25(6), 529-539.

Torra, V., \& Narukawa, Y. (2009, August). On hesitant fuzzy sets and decision. In Fuzzy Systems, 2009. FUZZ-IEEE 2009. IEEE International Conference on (pp. 1378-1382). IEEE.

Wang, F., Li, X., \& Chen, X. (2014). Hesitant fuzzy soft set and its applications in multicriteria decision making. Journal of Applied Mathematics, 2014, Article ID 643785, 10 pages.

Xia, M., \& Xu, Z. (2011). Hesitant fuzzy information aggregation in decision making. International Journal of Approximate Reasoning, 52(3), 395-407.

$\mathrm{Xu}, \mathrm{Z}$. (2016). Hesitant fuzzy sets theory. Springer.

Zadeh, L. A. (1965). Fuzzy sets. Information and Control, 8(3), 338-353.

Zhang, Z. (2013). Hesitant fuzzy power aggregation operators and their application to multiple attribute group decision making. Information Sciences, 234, 150-181. 